Proc. Indian Acad. Sci. (Chem. Sci.), Vol. 102, No. 2, April 1990, pp. 131-146.

C. Printed in India.

\title{
Singlet quenching of tetraphenylporphyrin and its metal derivatives by iron(III) coordination compounds
}

\author{
FRANCIS D'SOUZA and V KRISHNAN* \\ Department of Inorganic and Physical Chemistry, Indian Institute of Science, Bangalore \\ 560012 , India \\ MS received 6 November 1989; revised 6 January 1990
}

\begin{abstract}
The quenching of fluorescence of the free-base tetraphenylporphyrin, $\mathrm{H}_{2} \mathrm{TPP}$, and its metal derivatives, MgTPP and $\mathrm{ZnTPP}$ by diverse iron(III) complexes, $\left[\mathrm{Fe}(\mathrm{CN})_{6}\right]^{3-}$, $\mathrm{Fe}(\mathrm{acac})_{3},\left[\mathrm{Fe}(\mathrm{mnt})_{2}\right]^{-}, \mathrm{Fe}(\mathrm{Salen}) \mathrm{Cl},\left[\mathrm{Fe}_{4} \mathrm{~S}_{4}(\mathrm{SPh})_{4}\right]^{2-}, \mathrm{FeTPPCl}$ and $\left[\mathrm{Fe}(\mathrm{Cp})_{2}\right]^{+}$has been studied both in homogeneous medium $\left(\mathrm{CH}_{3} \mathrm{CN}\right)$ and micellar media, SDS, CTAB and Triton $X-100$. The quenching efficiencies are analysed in terms of diffusional encounters and it has been possible to separate static quenching components. The quenching constants are dependent on the nature of the ligating atoms around iron(III) and also on the extent of $\pi$-conjugation of the ligands. The quenching mechanism has been investigated using steady-state irradiation experiments. Evidence for oxidative quenching by iron(III) complexes was obtained, though the spin multiplicities of the excited electronic states of iron(III) complexes permit both energy and electron transfer mechanisms for quenching of the singlet excited state of the porphyrins.
\end{abstract}

Keywords. Fluorescence quenching; porphyrins; iron(III) coordination compounds.

\section{Introduction}

The photochemistry and photophysics of porphyrins and its metal derivatives are subjects of current interest. The relevance of these studies lies in their potential applications in mimicking the photosynthetic functions and solar energy conversion. In recent years there has been considerable research activity in the study of light-induced electron transfer reactions employing metalloporphyrins as donors (Mauzerall 1978; McLendon et al 1985; Mayo et al 1986). It is recognized that for efficient conversion and storage of light energy, the donor and acceptor have to be compartmentalized subsequent to light-induced charge-separation so as to avoid any wasteful charge recombination reaction. Micellar systems offer a great advantage in this respect. There have been a number of studies concerning the interactions of porphyrins with diverse neutral organic acceptors in organized assemblies (Siggel et al 1987; Kalyanasundaram 1987). These studies involved the use of optical spectroscopy to follow photo-induced electron transfer reactions. A few studies employed EPR spectroscopy to arrive at the nature of light-induced electron transfer products (Willigen and Ebersole 1987).

The rates of light-induced electron transfer reactions in the intermolecular

* For correspondence 
donor-acceptor systems are primarily dependent on the redox states and the nature of the donor (HOMO) and acceptor (LUMO) orbitals. Transition metal complexes possess very interesting redox properties with well-defined electronic structures. Metal complexes undergo sequential one-electron redox processes that are highly reversible. Moreover, the redox reactions are a sensitive function of the nature of the ligand and the metal. Coordination compounds of iron(III) with sulphide ligands play an important role as terminal electron acceptors in many enzymatic reactions (Hall and Evans 1969; Herriott et al 1970; Averill and Orme 1979). It would be of interest to study the electron-acceptor properties of various iron(III) complexes to delineate the nature of the ligand environments in the photo-redox behaviour.

We report here on the singlet quenching of 5,10,15, 20-tetraphenylporphyrin ( $\mathrm{H}_{2}$ TPP) and its magnesium(II) and zinc(II) derivatives (MgTPP and $\mathrm{ZnTPP}$ ) by a series of iron(III) coordination compounds bearing different ligand systems (figure 1). The iron(III) complexes employed are: potassium ferricyanide, $\mathrm{K}_{3} \mathrm{Fe}(\mathrm{CN})_{6}$; tris(2, 4-pentanedianoto)iron(III), $\mathrm{Fe}(\mathrm{acac})_{3}$; tetrabutylammonium bis(maleonitriledithioloto)iron(III), $\left(\mathrm{Et}_{4} \mathrm{~N}\right)\left[\mathrm{Fe}(\mathrm{mnt})_{2}\right] ; \mathbf{N}, \mathrm{N}^{\prime}$-bis salicylideneethylenediamine iron(III) chloride, $\mathrm{Fe}(\mathrm{Salen}) \mathrm{Cl}$; tetrabutylammonium tetraphenyl mercapto- $\mu_{3}$-sulphido-iron cluster, $\left(\mathrm{Bu}_{4} \mathrm{~N}\right)_{2}\left[\mathrm{Fe}_{4} \mathrm{~S}_{4}(\mathrm{SPh})_{4}\right] ; 5,10,15,20$-tetraphenylporphyrinato iron(III) chloride, FeTPPCl; and ferricinium hexafluorophosphate, $\left[\mathrm{FeCp}_{2}\right] \mathrm{PF}_{6}$. Quenching properties are studied in homogeneous, methyl cyanide, cationic, CTAB, anionic, SDS and neutral Triton X-100 micellar media. These studies are helpful in bringing out the influence of micellar media in the quenching reactions. The present study focusses on: (i) the nature of the quenching process (energy transfer and/or electron transfer process), (ii) the dependence of the quenching mechanism on the nature of iron(III) complexes, and (iii) the importance of charged quenched species and the nature of the micellar media in governing the photo-excited reactions. Wherever possible, steady-state illumination experiments have been carried out to characterize the photoreaction products.

\section{Experimental}

\subsection{Materials}

meso-Tetraphenylporphyrin ( $\left.\mathrm{H}_{2} \mathrm{TPP}\right)$ and its divalent metal derivatives $\mathrm{MgTPP}$ and ZnTPP were prepared and purified according to the procedure described in the literature (Rothemund and Mennotti 1941; Dorough et al 1951). The iron(III) complexes have been synthesized and purified as follows. Tris(2,4-pentanedianoto) iron(III) was prepared and purified by recrystallisation in ethanol (Lintvedt and Kernitshy 1970) (m.p. 179 ). Bis(maleonitriledithiolato) iron(III) was prepared from disodium salt of bis-1,2-dimercapto-1,2-dicynoethene and iron(III) chloride hexahydrate according to the method described by Weiher et al (1964). The crystallized product has an m.p. of $280^{\circ}$. Bis( $N, N^{\prime}$-salicylidineethylenediamine)iron(III) chloride was synthesized using the procedure described by Gerlock et al (1966). Tetraphenylporphyrinato iron(III) chloride was synthesized from $\mathrm{H}_{2}$ TPP and iron(II) sulphate (Rothemund and Mennotti 1948). The purity of the product was checked by absorption spectra $\left[\mathrm{C}_{6} \mathrm{H}_{6}\right.$ (solvent)- $\lambda_{\max }(\log \varepsilon), 374 \mathrm{~nm}(4 \cdot 76), 418 \mathrm{~nm}$

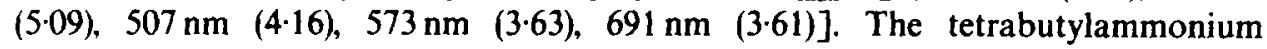
(tetraphenylmercapto- $\mu_{3}$-sulphido-iron) cluster was prepared from thiophenol, an- 
<smiles>CC1=C(C2CC2)SC2(S1)SC([Al])=C(C#N)S2</smiles>

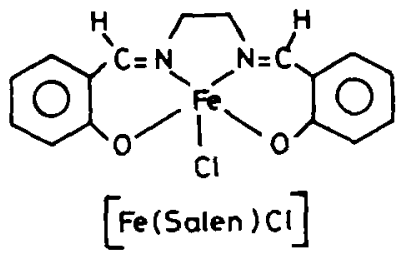

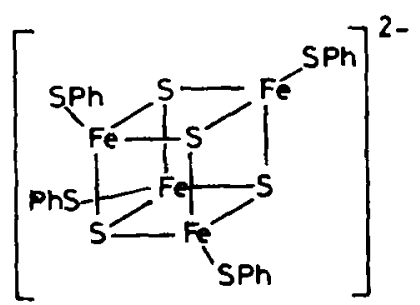<smiles>CC(C#N)(C#N)C#N</smiles><smiles>[Z]C1CC[As]1[AsH2]</smiles>

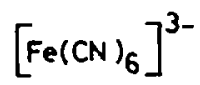<smiles></smiles><smiles>CC(C)(C)C(C)(C)F</smiles><smiles>c1ccc(-c2ccccc2)cc1</smiles>

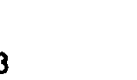

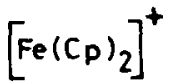

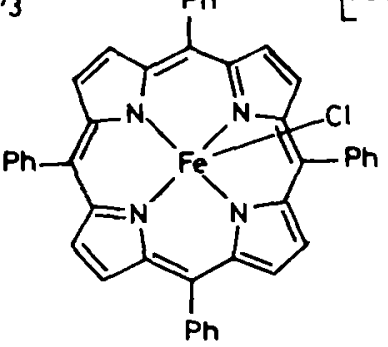

FeTPPCI

Figure 1. Structures of iron(III) coordination compounds used as acceptors.

hydrous iron(III) chloride and sodium hydrogen sulphide (Averill et al 1973) and it was purified by recrystallisation (m.p. 190-191 ${ }^{\circ}$ ). Ferricinium hexafluorophosphate was synthesized from cyclopentadienyl iron(II) according to the procedure described in the literature (Yang et al 1975). The purity of this compound was checked by absorption spectra $\left[\mathrm{CH}_{3} \mathrm{CN}\right.$ (solvent), in absence of air; $\left.\lambda_{\max }(\log \varepsilon), 620 \mathrm{~nm}(2 \cdot 64)\right]$. Potassium ferricyanide was obtained from BDH (India) and used as received.

The surfactants, sodium dodecyl sulphate (SDS), cetyltrimethyl ammonium bromide (CTAB) and Triton X-100 were procured from Sigma Chemicals, USA. SDS was purified by washing it with ether followed by recrystallisation in ethanol. All other 
chemicals and solvents were of reagent grade. Doubly distilled water was used throughout the work. The solvent $\mathrm{CH}_{3} \mathrm{CN}$ was dried and distilled before use.

\subsection{Methods}

Spectroscopic measurements were carried out with a Hitachi $3400 \mathrm{UV} /$ visible/near IR spectrophotometer. Emission intensities were monitored using a Hitachi (650-50) fuorescence spectrophotometer. The right angle detection method was employed. A Sonics and Materials Ultrasonicater was used for sonicating the solutions. A $250 \mathrm{~W}$ medium pressure mercury lamp and a halogen lamp $(250 \mathrm{~W})$ were used for steady-state photolysis experiments.

SDS and CTAB maintained at $30 \mathrm{mM}$ concentration and $3 \%(\mathrm{v} / \mathrm{v})$ Triton X-100 solutions were employed for micellar studies. The concentrations were well above the critical micellar concentration (cmc) values (Fendler 1982). Porphyrins, $\mathrm{H}_{2}$ TPP, MgTPP and ZnTPP were micellised using the following procedure. Stock solutions of porphyrins in $\mathrm{CH}_{2} \mathrm{Cl}_{2}$ were prepared by weighing appropriate quantities and dissolving them in $\mathrm{CH}_{2} \mathrm{Cl}_{2}$ to give $6 \mathrm{mM}$ concentrations. The concentrations were checked using the absorbance values. These solutions were diluted further to obtain a $0.5 \mu \mathrm{M}$ concentration of the porphyrins. A $10 \mathrm{ml}$ aliquot of this solution was solubilized in micelle and $\mathrm{CH}_{2} \mathrm{Cl}_{2}$ was allowed to evaporate by bubbling argon and slightly warming the mixture. A concentration ratio of [porphyrin]/[micelle] is maintained at $10^{-4}$ to ensure complete micellisation. The iron(III) coordination compounds were micellised using a similar procedure. To ensure complete and uniform distribution of the solubilizates, the mixtures were sonicated in pulses prior to recording the emission spectra.

Steady-state irradiation experiments were carried out either with a halogen lamp or a medium pressure mercury lamp. The light from the source is channelled through a three-compartment filter cell consisting of water (to filter UV radiation) and focussed on the sample through a lens. The test solutions were purged with argon gas throughout irradiation time. The absorption spectra of the test solutions were recorded periodically to monitor the nature of the reaction products.

\section{Results}

The electronic absorption spectral features of $\mathrm{H}_{2}$ TPP and its divalent metal derivatives, $\mathrm{MgTPP}$ and $\mathrm{ZnTPP}$ in homogeneous medium $\left(\mathrm{CH}_{3} \mathrm{CN}\right)$, remain unaltered in the different micellar media. The iron(III) complexes exhibit intense absorption bands in homogeneous and micellar media in the Soret region of absorption of porphyrins (figure 2). The absorbance values of Soret and $Q$ bands of porphyrins are not significantly altered on addition of iron(III) complexes in $\mathrm{CH}_{3} \mathrm{CN}$ and micellar media. Typically, addition of iron(III) complexes in $\mathrm{CH}_{3} \mathrm{CN}(1.2 \mathrm{mM})$ to a solution containing ZnTPP (0.5 mM) resulted in very little change in absorbance value $(0.01)$ of the $550-\mathrm{nm}$ band of porphyrin. This indicates that the association of porphyrins and iron(III) complexes is very weak $\left(K_{\text {ass }}<0.01 \mathrm{~m}^{-1}\right)$. It is of interest to note that the micellar media have no effect on the emission spectra of the porphyrins. Thus, the singlet emission bands of porphyrins, $\mathrm{H}_{2} \operatorname{TPP}(654,720 \mathrm{~nm}), \operatorname{MgTPP}(604,652 \mathrm{~nm})$ and $\mathrm{ZnTPP}(604,652 \mathrm{~nm})$ obtained on excitation at the respective Soret absorption 


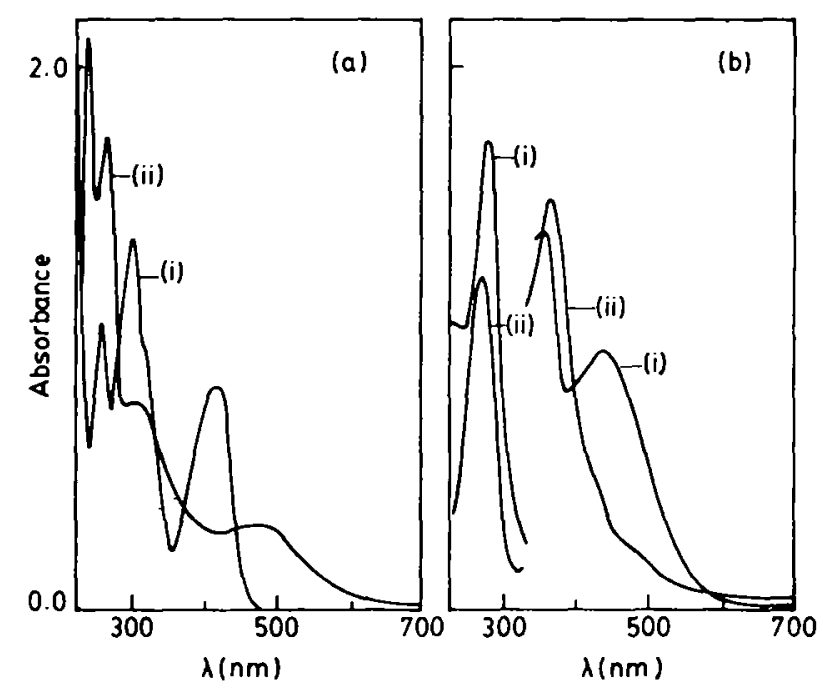

Figure 2. The electronic absorption spectra of (a)(i) $\mathrm{K}_{3} \mathrm{Fe}(\mathrm{CN})_{6}(1.8 \mathrm{mM})$; (ii) $\mathrm{Fe}(\mathrm{acac})_{3}$, $(0.26 \mathrm{mM})$; and (b)(i) $\left[\mathrm{Fe}(\mathrm{mnt})_{2}\right]^{-},(0.2 \mathrm{mM})$; (ii) Fe(Salen)Cl, $(0.28 \mathrm{mM})$ in SDS solutions. The spectra are expanded 4 times in the region $350-700 \mathrm{~nm}$ for clarity.

bands (418 nm for $\mathrm{H}_{2}$ TPP and $420 \mathrm{~nm}$ for $\mathrm{ZnTPP}$ and $\mathrm{MgTPP}$ ) in $\mathrm{CH}_{3} \mathrm{CN}$ are reproduced in the different micellar media.

The intensity of the fluorescence emission bands decreased on increasing addition of iron(III) complexes in different media (figure 3). The data obtained were analysed using the Stern-Volmer relation

$$
\begin{aligned}
I_{0} / I & =1+K_{\mathrm{sv}}[Q], \\
K_{\mathrm{sv}} & =k_{Q} \tau,
\end{aligned}
$$

where $I_{0}$ and $I$ denote the fluorescence intensity of porphyrins in the absence and presence of iron(III) complexes (quenchers, $[Q]$ ). It is necessary to correct for the emission intensity (I) of porphyrins, since the iron(III) complexes absorb strongly in the excitation wavelength (Soret band) of the porphyrins. This is carried out using the method of Demas and Adamson (1973). The corrected emission ratio $\left(I_{0} / I\right)_{\text {corr }}$ is calculated from the relation

$$
\left(I_{0} / I\right)_{\text {corr }}=\left(I_{0} / I\right)_{\text {obsd }}\left[\frac{1-10^{-(\mathrm{Ad}-\mathrm{Aq})}}{1-10^{-\mathrm{Ad}}}\right]\left[\frac{A_{d} 10^{-\mathrm{Aq}^{\prime}}}{A_{d}+A_{q}}\right],
$$

where $\left(I_{0} / I\right)_{\text {obs }}$ is the observed ratio of luminescence intensities and $\left(I_{0} / I\right)_{\text {corr }}$ is the ratio of intensities corrected for absorption of the incident light by the quencher and for the trivial absorption of the emitted light by the solution. $A_{d}$ and $A_{q}$ are the absorbance values per unit path length at the exciting wavelength for the donor and quencher respectively. The absorbance per unit path length of the quencher at the emitting wavelength is represented by $\boldsymbol{A}_{q}^{\prime}$.

The $K_{\mathrm{sv}}$ values obtained from the plots of $\left(I_{0} / I\right)_{\text {corr }}$ versus [Q] are found to be high in magnitude $\left(10^{2}-10^{3}\right)$. In addition, the plots reveal an upward curvature for the 

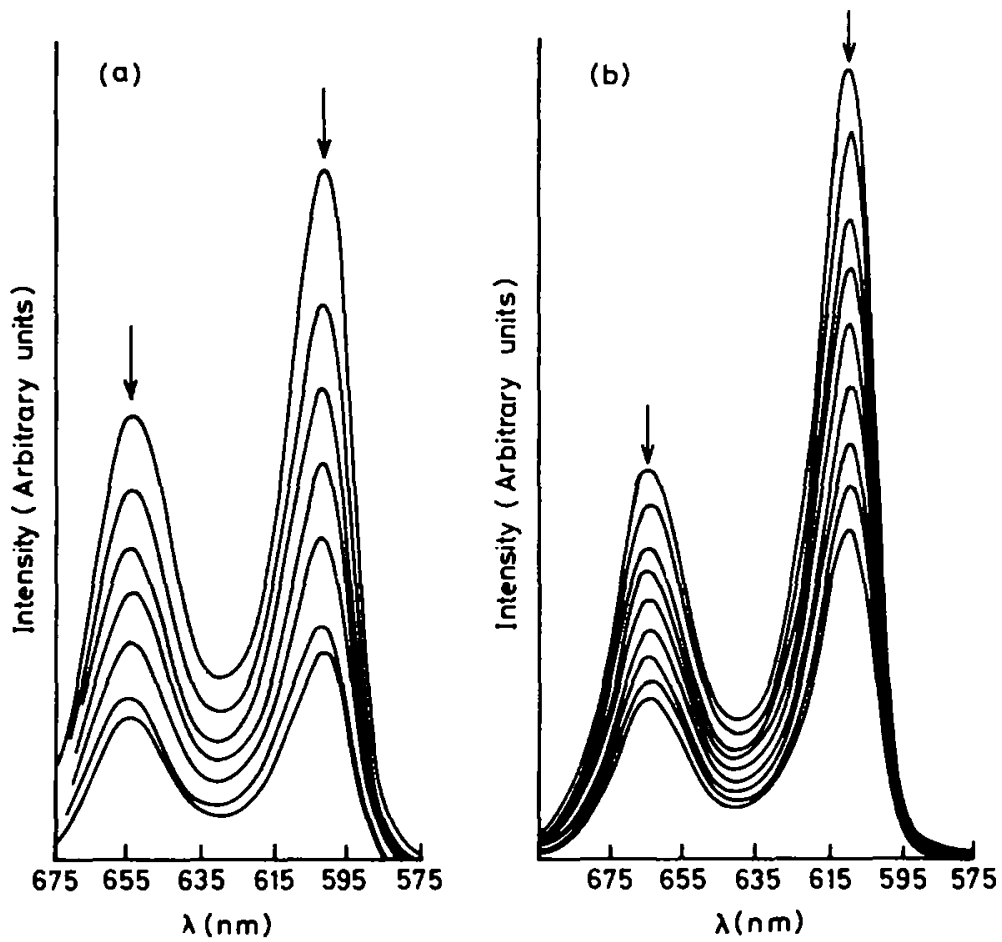

Figure 3. The quenching of the fluorescence bands of (a) ZnTPP on increasing addition of $\mathrm{Fe}(\mathrm{Salen}) \mathrm{Cl}\left([Q]=0-1-0.6 \times 10^{-3} \mathrm{M}\right)$, and (b) MgTPP on increasing addition of $\left[\mathrm{Fe}(\mathrm{mnt})_{2}\right]^{-}$ $\left([Q]=0.1-0.8 \times 10^{-3} \mathrm{M}\right)$ in Trition X-100. (i iex $\left.=420 \mathrm{~nm}\right)$.

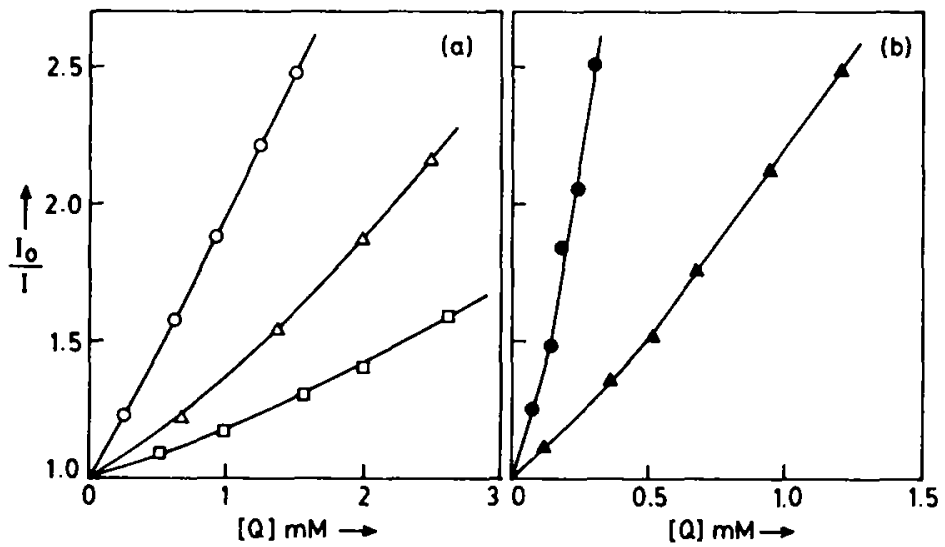

Figure 4. Stern-Volmer plots of (a) $(\triangle) \mathrm{MgTPP}-\mathrm{Fe}($ Salen $) \mathrm{Cl},(\mathrm{O}) \mathrm{MgTPP}-\mathrm{Fe}(\mathrm{acac})_{3},(\square)$ $\mathrm{MgTPP}_{-}\left[\mathrm{Fe}_{4} \mathrm{~S}_{4}(\mathrm{SPh})_{4}\right]^{2-}$, and (b) $(\Delta) \mathrm{MgTPP}_{-}\left[\mathrm{Fe}(\mathrm{mnt})_{2}\right]^{-}$, (O) $\mathrm{MgTPP}_{\mathrm{g}} \mathrm{FeTPPCl}$. The fluorescence intensities are corrected for absorption of the quencher in the excitation wavelength region.

different iron(III) complexes (figure 4). Deviation from the expected linearity could be accounted for by the fact that only a certain fraction of the excited state is actually involved in collisional quenching. Moriya (1984) has proposed a model to correct for 
these deviations. Assuming that the quenching reactions are diffusion-controlled and introducing a transient term in Smoulchowski's equation, one can estimate the actual fraction of molecules of the excited state involved in collisional quenching process. This can be expressed as (Roy and Mukherjee 1988),

$$
\frac{1-\left(I / I_{0}\right)_{\text {corr }}}{[Q]}=K_{\mathrm{sv}}\left(I / I_{0}\right)_{\mathrm{corr}}+\frac{1-W}{[Q]},
$$

where $W$ represents the fraction of donor molecules in the excited state that are actually quenched by collisional mechanism. The fraction $1-W$ denotes the donor molecules in the excited state which are deactivated as soon as formed. Plots of $\left[1-\left(I / I_{0}\right)_{\text {corr }}\right] /$ $[Q]$ versus $\left(I / I_{0}\right)_{\text {cors }}$ were made for obtaining $K_{\text {sv }}$ values corrected for static quenching (figure 5). The intercepts of these plots in the ordinate axis $\left[\left(I / I_{0}\right)_{\text {corr }} \rightarrow 0\right]$ permit evaluation of the values of $W$. The data obtained are subjected to linear least-squares analysis. The range of values of $W$ for different $[Q]$ values was calculated. The results of quenching experiments are presented in table 1 . It may be mentioned here that changing the excitation wavelength from the Soret absorption band to the visible absorption region of the porphyrins $(\hat{\lambda}=550 \mathrm{~nm})$ does not produce any significant change in the $K_{\mathrm{sv}}$ values.

Examination of table 1 leads to the following observations: (i) The $K_{\mathrm{sv}}$ values for different quenchers with reference to a given porphyrin decrease in the order $\mathrm{Fe}($ Salen $) \mathrm{Cl}<\left[\mathrm{Fe}_{4} \mathrm{~S}_{4}(\mathrm{SPh})_{4}\right]^{2-}<\mathrm{Fe}(\mathrm{acac})_{3}<\left[\mathrm{Fe}(\mathrm{mnt})_{2}\right]^{-}<\mathrm{FeTPPCl}$. (ii) The magnitude of quenching constant values for different donor porphyrins varies as $\mathrm{ZnTPP}<\mathrm{MgTPP}<\mathrm{H}_{2} \mathrm{TPP}$, irrespective of the nature of the quenchers. (iii) The $K_{\mathrm{sv}}$ values differ in going from homogeneous to micellar media depending on the nature

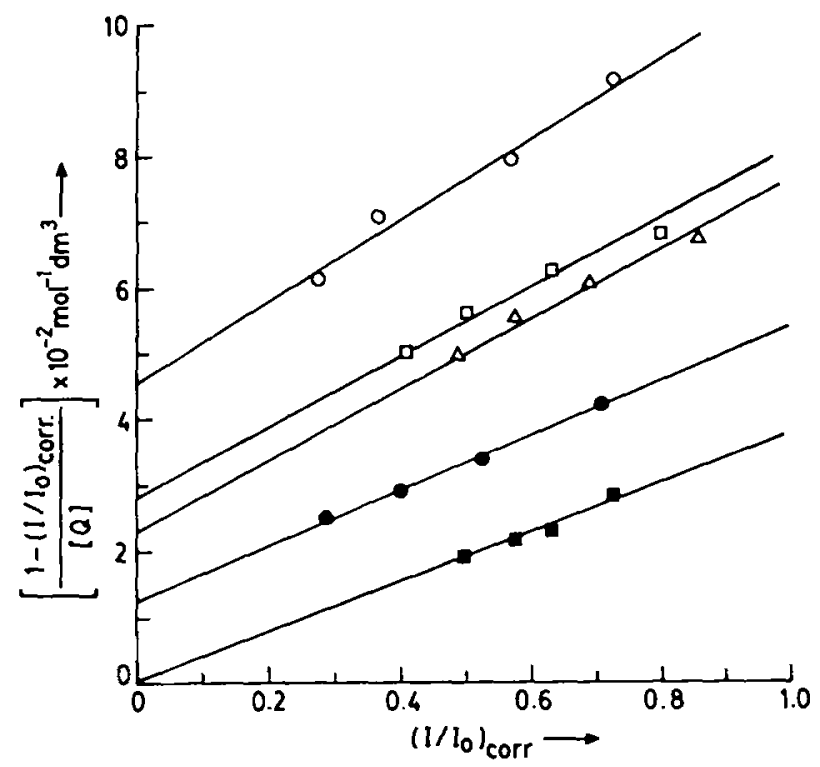

Figure 5. Plots of $\left[1-\left(I / I_{0}\right)_{\text {corr }}\right] /[Q]$ versus $\left(I_{0} / I\right)_{\text {corr }}$ of MgTPP-Fe(III) complexes in $\mathrm{CH}_{3} \mathrm{CN}$ at $25^{\circ} \mathrm{C}$. (O), Fe(Salen)Cl; $(\square)$, Fe(acac) $)_{3} ;(\triangle)$, [Fe(mnt) $]^{-} ;(\mathrm{O})$, FeTPPCl; (回), $\left[\mathrm{Fe}_{4} \mathrm{~S}_{4}(\mathrm{SPh})_{4}\right]^{2-}$. The values on the $\mathrm{Y}$-axis have to be multiplied by 2 and 5 for [Fe(mnt $\left.)_{2}\right]^{-}$ and $\mathrm{FeTPPCl}$ respectively. 


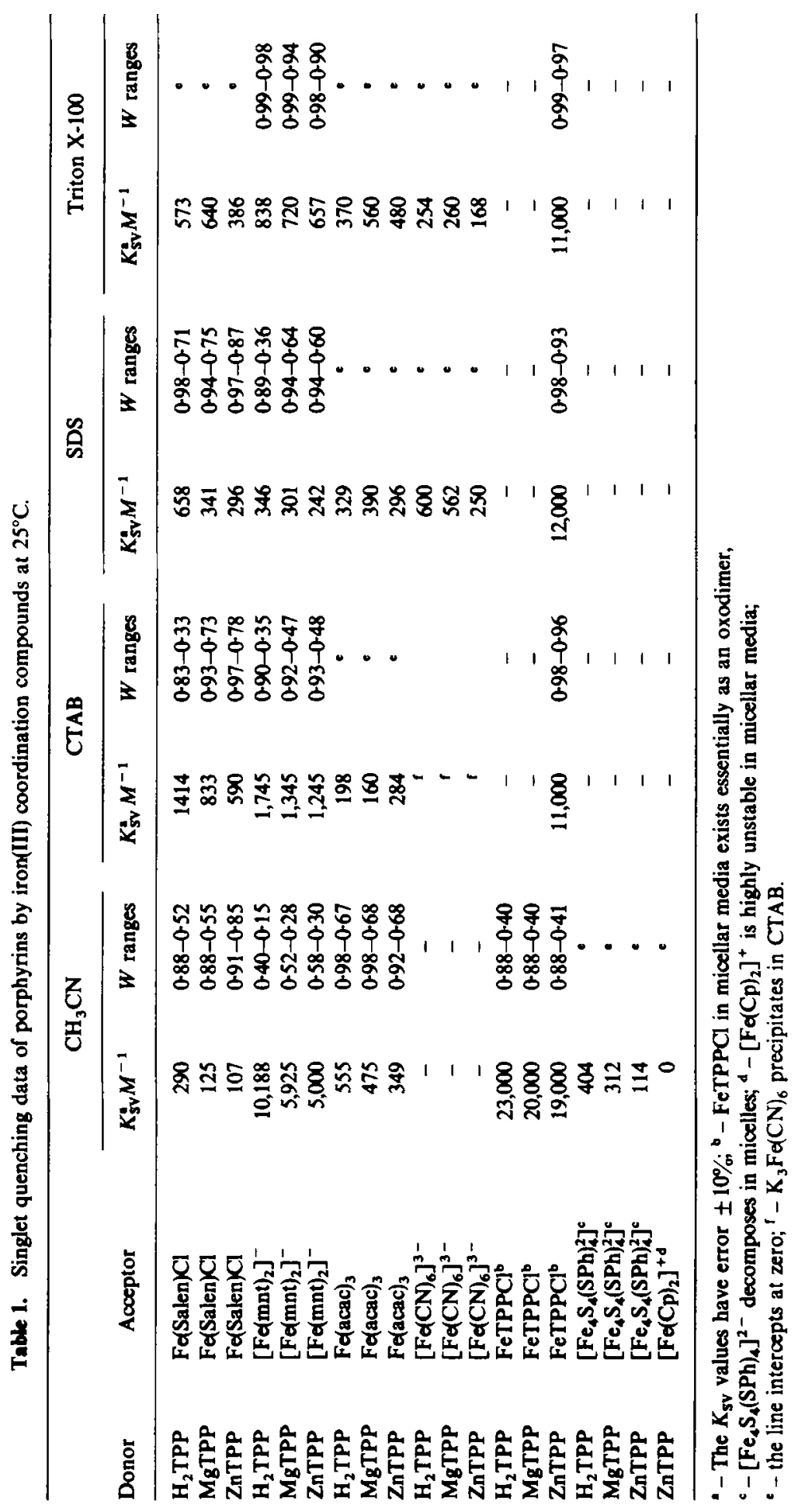


of the quenchers. For the neutral quencher, $\mathrm{Fe}(\mathrm{Salen}) \mathrm{Cl}$, the $K_{\mathrm{sv}}$ values are found to be lower in homogeneous medium relative to that in micellar media whilst for FeTPPCl and $\mathrm{Fe}(\mathrm{acac})_{3}$, higher values of quenching constant are obtained in the homogeneous medium relative to those in different micellar media. It is difficult to rationalize the influence of cationic, anionic and neutral micellar media on the $K_{\mathrm{sv}}$ values obtained in this study. The expectation that a negatively charged quencher would exhibit enhanced bimolecular quenching in cationic micelles is borne out only in the case of $\left[\mathrm{Fe}(\mathrm{mnt})_{2}\right]^{-}$. The quenching constant values obtained for $\mathrm{FeTPPCl}$ in micellar media are large in magnitude relative to that obtained in the homogeneous medium. This can be attributed to the presence of iron $\mu$-oxodimer (Buchler 1975), (FeTPP) ${ }_{2} \mathrm{O}$. (iv) The range of $W$ values obtained for the different systems varies markedly with respect to the nature of the quencher and the medium. The upper limit in most of the cases is in the neighbourhood of unity while the lower limit ranges from 0.3 to 0.4 . It is worthy of mention here that $\left[\mathrm{Fe}(\mathrm{mnt})_{2}\right]^{-}$is the only quencher in Triton X-100 which exhibits a near-unity $W$ value while the other quenchers have a zero intercept in the same medium. The differing magnitudes of quenching constants in various media with the diverse nature of the quenchers are useful in arriving at the possible mechanisms of the fluorescence quenching process.

Interesting results are obtained in steady-state irradiation experiments involving metalloporphyrins and different iron(III) complexes. The optical absorption spectra of an irradiated sample containing a mixture of a metalloporphyrin MgTPP or ZnTPP $(0.01 \mathrm{mM})$ and $\mathrm{Fe}(\mathrm{acac})_{3}(0.05 \mathrm{mM})$ in $\mathrm{CH}_{3} \mathrm{CN}$ revealed bleaching of Soret and $Q$ bands of porphyrins accompanied by the appearance of new bands at 760 and $850 \mathrm{~nm}$. The latter is indicative of the formation of an isoporphyrin (Dolphin et al 1970). The time course of this reaction is shown in figure 6. Steady state irradiation

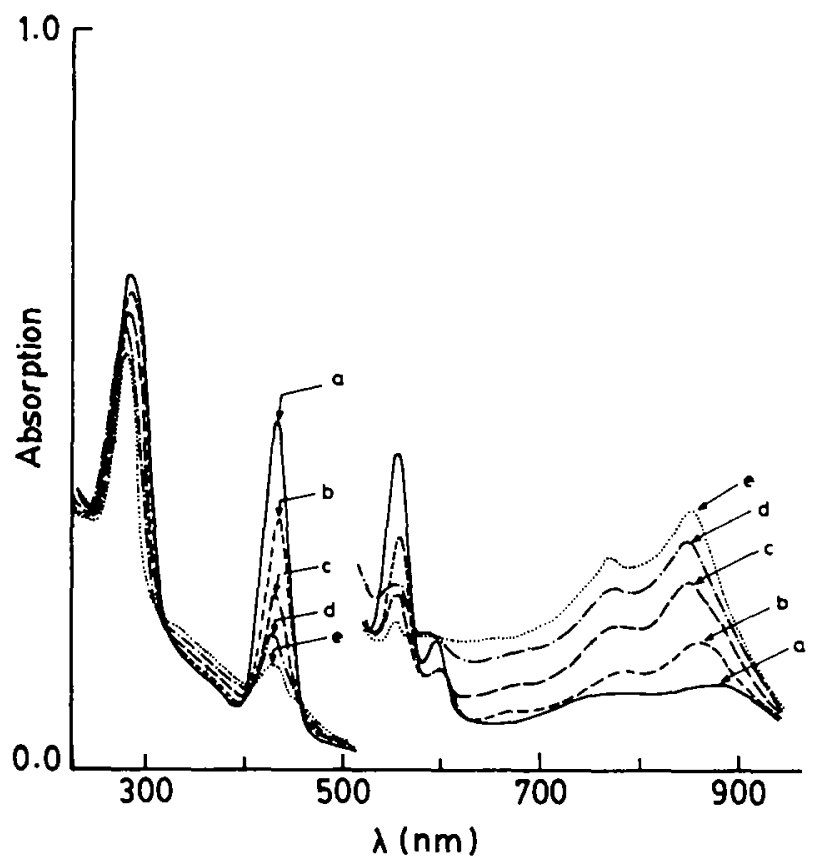

Figure 6. The electronic absorption spectra of $\mathrm{ZnTPP}$ containing $\mathrm{Fe}(\mathrm{acac})_{3}$ in $\mathrm{CH}_{3} \mathrm{CN}$ at different periods of irradiation. (a) 0 , (b) 10 , (c) 20 , (d) 30 , and (e) $40 \mathrm{~min}$. The $Y$-axis in the spectra at the longer wavelength region $(500-900 \mathrm{~nm})$ is expanded for clarity. 
of porphyrins alone in the solvent and micellar media, however, does not produce isoporphyrin. In order to probe into the mechanism of formation of isoporphyrin, irradiation experiments were carried out in argon and $\mathrm{N}_{2} \mathrm{O}$ atmospheres. It is found that $\mathrm{N}_{2} \mathrm{O}$ completely inhibits the formation of isoporphyrin. Interestingly, irradiation experiments performed in the presence of sacrificial donors such as EDTA or $o$-phenanthroline produced no isoporphyrin. Attempts were made to characterize the oxidation products using NMR and mass spectral data. In view of the small amount of the products obtained in the irradiation experiments and the high molecular weight of isoporphyrin with different functionalities, it was not possible, however, to arrive at the structure of the products. Hence more reliance is placed on the optical absorption data. In addition to the appearance of a long wavelength band at $850 \mathrm{~nm}$, characteristic of isoporphyrin formation, it is found that the absorption bands of $\mathrm{Fe}(\mathrm{acac})_{3}$ in the $U \mathrm{~V}$-visible region decreased in intensity with the progress of irradiation. In order to avoid any thermal reactions during irradiation, samples were cooled. The results obtained from these experiments demonstrate the formation of isoporphyrin accompanied by reduced ferrous ions. It is interesting to note that similar experiments performed using free-base porphyrin $\mathrm{H}_{2} \mathrm{TPP}$, however, produced no isoporphyrins. Moreover, irradiation experiments on mixtures of porphyrins and other quenchers showed only very small changes in the intensity of Soret and $Q$ bands of porphyrins. It may be mentioned that $\left[\mathrm{Fe}_{4} \mathrm{~S}_{4}(\mathrm{SPh})_{4}\right]^{2-}$ and $\left[\mathrm{FeCp}_{2}\right]^{+}$decomposed under similar irradiation conditions. We failed to detect any one-electron reduced products in the reactions involving other quenchers, possibily because of the instability of such photo-reduced products. In all the cases, experiments were conducted by irradiating the donor and acceptor separately to avoid any artefacts. Similar results are obtained in micellar media, however, with reduced yield of photo-reaction products. The data obtained provide probes into the mechanistic details of the quenching process.

\section{Discussion}

The results obtained in this study show that the iron(III) complexes efficiently quench the fluorescence of $\mathrm{H}_{2}$ TPP, MgTPP and $\mathrm{ZnTPP}$. The different channels by which the singlet excited state of the porphyrins can decay are: (a) complexation of the excited singlet state of porphyrin with iron(III) coordination compounds, (b) excited

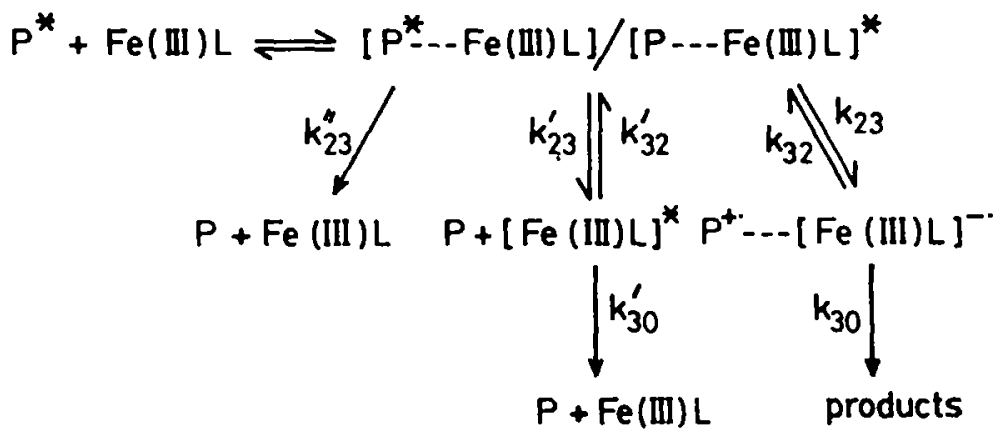

(a)

(b)

(c)

Scheme 1. 
energy transfer from porphyrin to the iron(III) complexes, and (c) oxidative electron transfer from excited porphyrins to the iron(III) complexes. These mechanisms are represented in the scheme 1 , where $P^{*}$ denotes the singlet excited state of the porphyrins ( $\mathrm{H}_{2}$ TPP, MgTPP and ZnTPP), and Fe(III)L stands for Fe(III) coordination compounds with $\mathrm{L}$ being the ligand. The fluorescence quenching constants $\left(K_{\mathrm{sv}}\right)$ obtained in this study are used to calculate the rate of bimolecular quenching constant $\left(k_{Q}\right)$ from the known life time of the porphyrin (table 2). The derived $k_{Q}$ values exceed the diffusion control limit possibly due to incomplete correction for ground state complexation. However, the $k_{Q}$ values show interesting variations with the nature of the different quenchers.

The $k_{Q}$ values are found to depend on the nature of the ligand, the stereochemistry of iron(III) in the complexes and the electronic structure of the coordination compounds. The spectral transitions of iron(III) complexes (table 3) (Lever 1984) are useful in arriving at the possible mechanism of the fluorescence quenching process. The paramagnetic complexes employed in this study are both of high-spin $\left(t_{2 \theta}^{3} e_{\theta}^{2} ;{ }^{2} A_{1}\right)$ and low spin $\left(t_{2 g}^{5} ;{ }^{2} A_{2}\right)$ types. The presence of unpaired electrons in the iron(III) complexes can deactivate the excited singlet state of the porphyrin to the triplet state. This is a trivial mechanism of fluorescence quenching of the porphyrins. The optical transitions in these complexes are dominated by intense LMCT and MLCT transitions $\left(t_{2} \rightarrow \pi^{*}\right),\left(\pi \rightarrow t_{2}^{*}\right)$, while the $d-d$ transitions in these complexes occur as shoulders in the neighbourhood of CT transitions and are often difficult to assign. These transitions involve excited state quartets and doublets. The spin restrictions permit only a few states for consideration of energy- and electron-transfer quenching. According to the Wigner spin rule, the spin-allowed transitions are only those with a spin multiplicity doublet (2) in the case of doublet $\left({ }^{2} T_{2},{ }^{2} E_{2}\right)$ electronic ground state complexes and sextet (6), quartet (4) and doublet (2), in the case of sextet $\left({ }^{6} A_{1}\right)$ ground state of Fe(III) complexes. The excited-energy transfer from porphyrins to the sextet, quartet or doublet states of iron(III) complexes appears to be the possible mechanism for fluorescence quenching of porphyrins. However, it is observed that all the Fe(III) complexes except $\left[\mathrm{FeCp}_{2}\right]^{+}$do not absorb in the emission region $(\lambda>600 \mathrm{~nm})$ of the porphyrins. Moreover, the iron(III) complexes do not possess any emission bands in the region of porphyrin absorption. This indicates that energy transfer is not a likely mechanism for fluorescence quenching though direct proof for the absence of this mechanism is difficult to arrive at.

It is worthwhile considering the excited state electron transfer reaction in these systems as a source of fluorescence quenching. The electrochemical redox data of Fe(III) complexes serve to arrive at the thermodynamic criterion for the feasibility of the electron transfer reaction. Using the expression

$$
\Delta G_{23}^{0}=E_{1 / 2}\left(P^{+} \cdot / P\right)+E_{1 / 2}(\mathrm{Fe}(\mathrm{III}) \mathrm{L} / \mathrm{Fe}(\mathrm{II}) \mathrm{L})-E_{(S, T)},
$$

and substituting the singlet or triplet energies of donor porphyrins, it is possible to obtain the free energy change for the electron transfer reaction, originating either from the singlet $(S)$ or triplet $(T)$ states. The singlet and triplet energies of $\mathrm{H}_{2}$ TPP, ZnTPP, MgTPP used in this calculation are $1 \cdot 86,2 \cdot 05,2.03 \mathrm{eV}$ for the singlet state, and $1.45,1.59,1.57 \mathrm{eV}$ for the triplet state, respectively (Seely 1978). The $\Delta G_{23}^{0}$ values are calculated for the singlet excited state of the porphyrins and are given in table 2. It is found that the $\Delta G_{23}^{o}$ values for electron transfer reactions from the singlet excited states are relatively more exergonic compared to those obtained from the triplet states. 


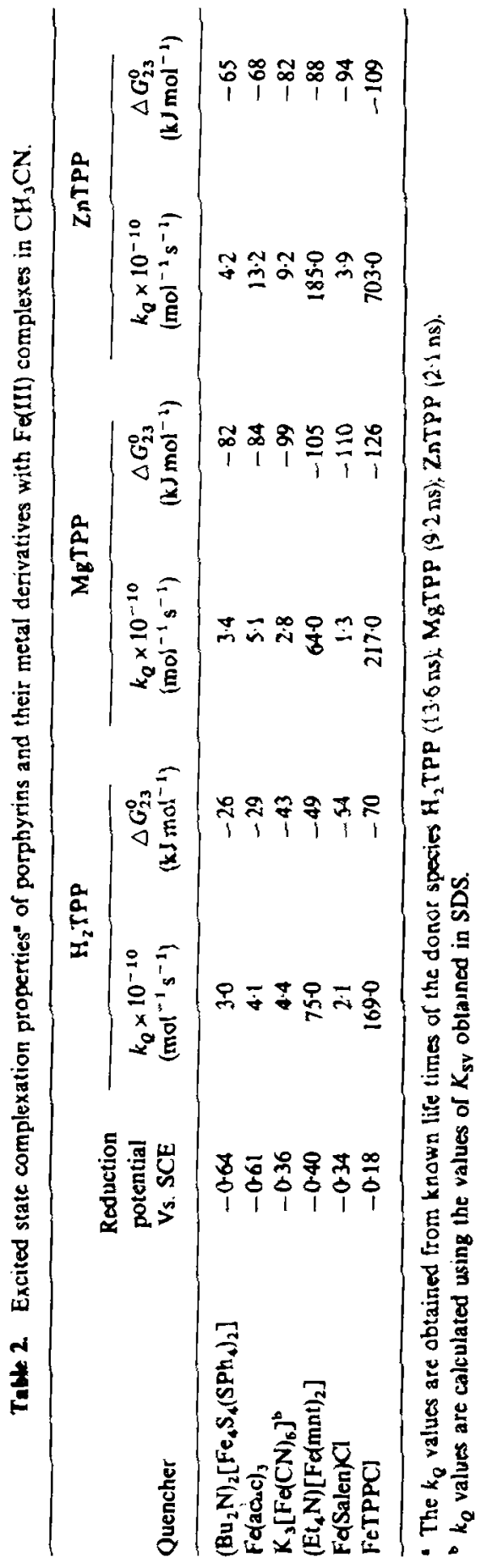


Table 3. Electronic absorption transitions of Fe(IIl) complexes".

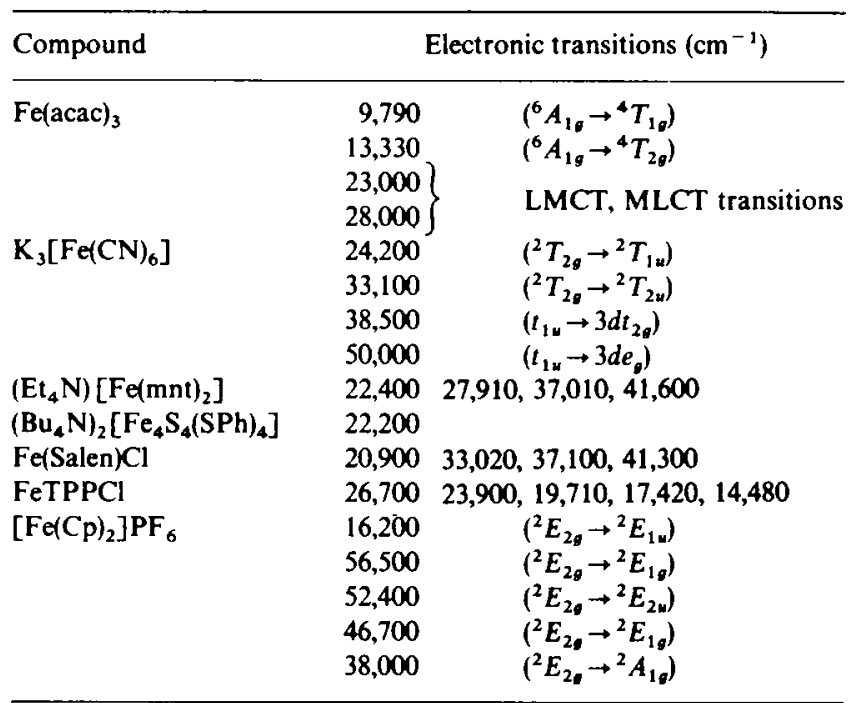

The data given are in $\mathrm{CH}_{3} \mathrm{CN}$ except in the case of ferricyanide where aqueous solution is used.
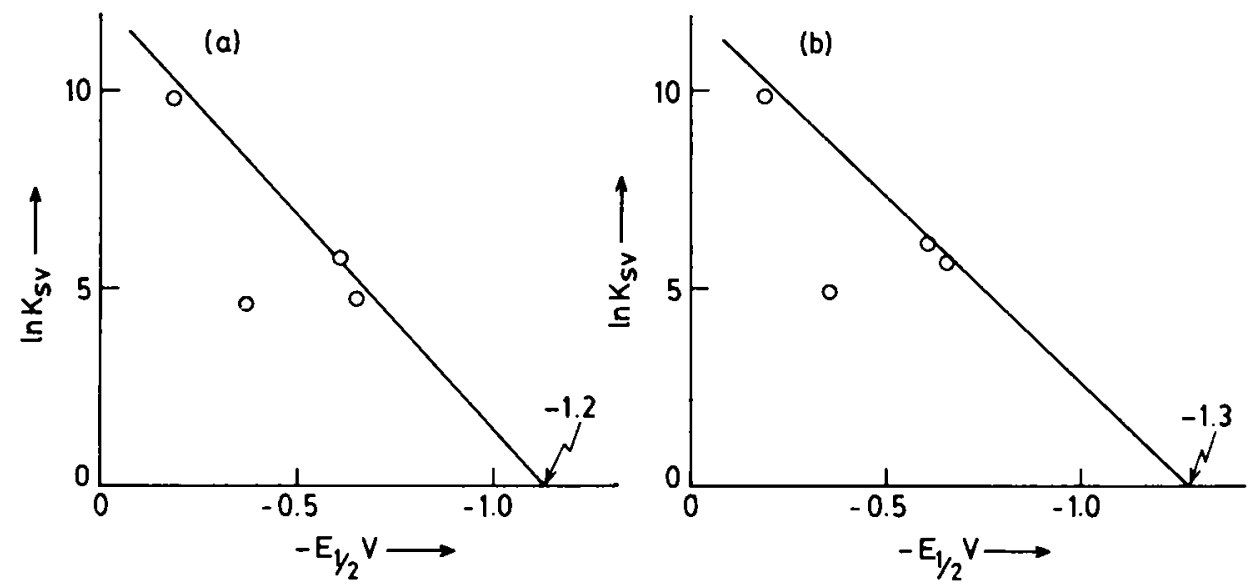

Figure 7. Plots of $\ln K_{\mathrm{sv}}$ versus $E_{1 / 2}$ values of the various acceptors for the quenching of (a) ZnTPP, and (b) MgTPP in $\mathrm{CH}_{3} \mathrm{CN}$.

A plot of $\ln K_{\mathrm{sv}}$ versus $E_{1 / 2}\left(\mathrm{FeL} / \mathrm{FeL}^{-}\right)$(reduction potentials of the quenchers) is linear, with the intercept on the $X$-axis representing the reduction potential of the singlet excited state of the donor (figure 7). The excited state reduction potentials of the donor porphyrins $\left(\mathrm{H}_{2} \mathrm{TPP}^{+} \cdot / \mathrm{H}_{2} \mathrm{TPP}^{*}, \mathrm{MgTPP}^{+} \cdot / \mathrm{MgTPP}^{*}\right.$ and $\mathrm{ZnTPP}^{+} \cdot / \mathrm{ZnTPP}^{*}$ ) obtained from these plots are $-1 \cdot 0,-1 \cdot 2,-1 \cdot 3 \mathrm{~V}$ for $\mathrm{H}_{2} \mathrm{TPP}$, $\mathrm{ZnTPP}$ and MgTPP respectively. These values agree well with the computed value of excited state reduction potential using the relation

$$
E_{1 / 2}\left(\mathrm{P}^{+} \cdot / \mathrm{P}^{*}\right)=E_{1 / 2}\left(\mathrm{P} / \mathrm{P}^{+\cdot}\right)-E_{S(0-0)},
$$


where $\mathrm{P}, \mathrm{P}^{*}$ and $\mathrm{P}^{+}$. represent the porphyrin ground state, singlet excited state of the donor and oxidized donor respectively. This suggests that the excited electron transfer is a likely mechanism for quenching of the excited state by $\mathrm{Fe}$ (III) complexes.

The rates of bimolecular quenching can be related to $\Delta G_{23}^{0}$ values according to Rehm and Weller (1970),

$$
k_{Q} \simeq \frac{k_{12} k_{30}}{2 k_{21}} \exp \left(\frac{-\Delta G_{23}^{0}}{R T}\right) .
$$

Plots of $\log k_{Q}$ versus $\Delta G_{23}^{0}$ are shown in figure 8. This indicates that the increase in $k_{Q}$ values in the weakly exergonic region is somewhat linear and rises rapidly in the moderate exergonic region, and finally reaches a limiting value. The rate is expected to decrease with increasing exothermicity for highly exothermic reactions (Marcus 1965). Reactions with large exergonicities have not been possible in the present study since metalloporphyrins are known to undergo oxidation reactions with acceptors having high reduction potentials (Fuhrhop and Mauzerall 1969). The non-linear relationship is very likely to arise from the possibility of the ion-pair having a low-lying electronically excited state (Miller et al 1984). Initially the electron is transferred to this state $\left(\pi^{*} \mathrm{CT}\right)$ rather than to the ground state directly.

The steady-state photolysis experiments offer valuable evidence in support of the electron-transfer mechanism. In what follows we shall discuss the results obtained from these measurements. It has earlier been demonstrated from EPR studies that ferricyanide quenching of porphyrin fluorescence is followed by the electron-transfer mechanism (Willigen and Ebersole 1987). Usage of $\mathrm{Fe}(\mathrm{acac})_{3}$ as a quencher clearly demonstrates the existence of electron transfer from the singlet excited state of

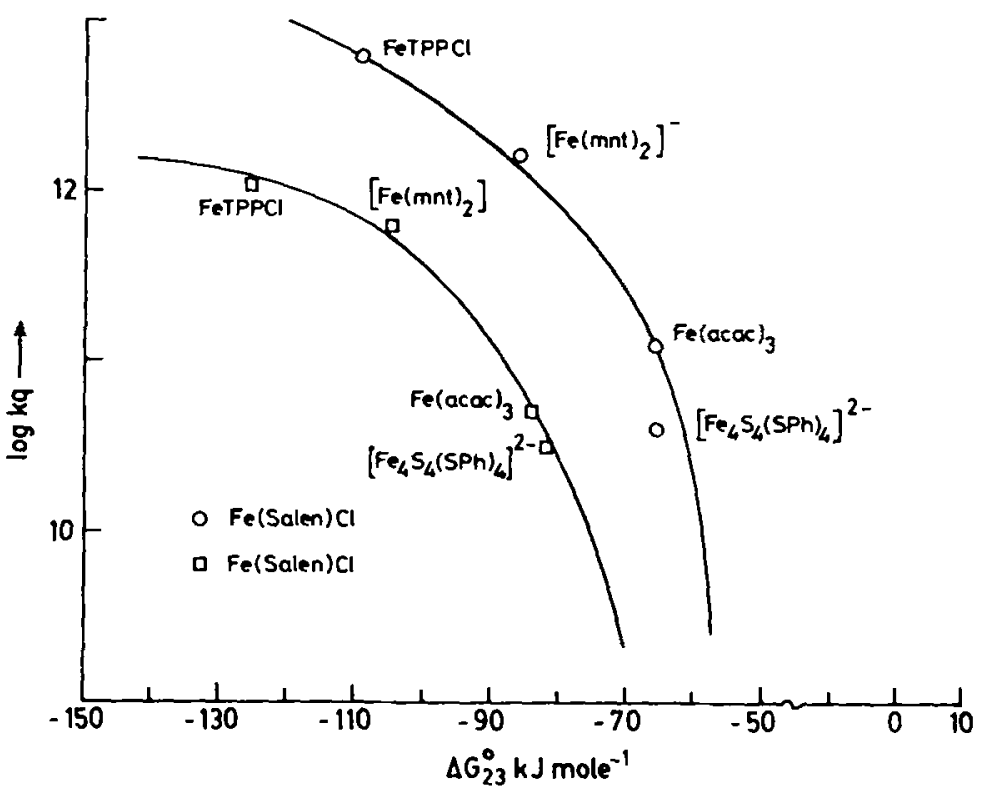

Figure 8. Plots oi $\log k_{Q}$ versus $\Delta G_{23}^{0}$ for the singlet emission quenching of ZnTPP $(\mathrm{O}--\mathrm{O})$ and $\mathrm{MgTPP}(\square---\square)$ in $\mathrm{CH}_{3} \mathrm{CN}$ at $25^{\circ} \mathrm{C}$. 
porphyrin. The results obtained here permit us to suggest the following scheme of reactions for the formation of isoporphyrin:

$$
\begin{aligned}
& \text { MTPP } \stackrel{h v}{\longrightarrow} \mathrm{MTPP}^{*}(\mathrm{M}=\mathrm{Zn} \text { or } \mathrm{Mg}) \\
& \mathrm{MTPP}^{*}+\left[\mathrm{Fe}(\mathrm{acac})_{3}\right] \longrightarrow \mathrm{MTPP}^{+}+\left[\mathrm{Fe}(\mathrm{acac})_{3}\right]^{-} \\
& 2 \mathrm{MTPP}^{+} \cdot \longrightarrow \mathrm{MTPP}^{+}+\mathrm{MTPP} \\
& {\left[\mathrm{Fe}(\mathrm{acac})_{3}\right]^{-} \longrightarrow\left[\mathrm{Fe}(\mathrm{acac})_{2}\right]+\mathrm{acac}^{-} \text {. }} \\
& \mathrm{MTPP}^{2+}+\mathrm{acac}^{-\cdot} \longrightarrow \text { isoporphyrin }+ \text { products } \\
& \mathrm{MTPP}^{2+}+\text { solv } \rightarrow \text { isoporphyrin }+ \text { products }
\end{aligned}
$$

The 'solv' represents water in the case of micellar media. It is found that the electrochemically produced monocation radical of porphyrin does not react with $\mathrm{Fe}(\mathrm{acac})_{3}$ to produce isoporphyrin. Further, the electronic absorption spectra of reaction mixtures during the course of irradiation do not show any bands characteristic of MTPP ${ }^{+.}$'s. Moreover, addition of sacrificial donors like EDTA inhibits the formation of isoporphyrin. This suggests the existence of bipositive $\mathrm{MTPP}^{2+}$ in solution. The presence of reduced species of $\left[\mathrm{Fe}(\mathrm{acac})_{3}\right]$ is clearly seen as a phenanthroline complex in the absorption spectra of the photolysed products $\left[\lambda_{\max }=440 \mathrm{~nm}\right)$. That the reactions leading to the formation of isoporphyrins is through a free radical mechanism is clearly manifested by the total inhibition of these reactions in the presence of the free-radical scavenger $\mathrm{N}_{2} \mathrm{O}$. We also eliminate any possibility of intermediacy of singlet oxygen by conducting the experiments in the presence of argon gas. Arguably, $\mathrm{MTPP}^{2+}$ is a strong electrophile and thus any nucleophile such as acac ${ }^{-}$or solv $/ \mathrm{H}_{2} \mathrm{O}$ can attack the porphyrin to form isoporphyrin. Presently we are unable to clarify whether the MTPP $^{2+}$ is attacked by the anion radical $\left(\mathrm{acac}^{-} / \mathrm{OH}^{-}\right)$) or the anion $\left(\mathrm{acac}^{-}\right.$or $\left.\mathrm{OH}^{-}\right)$. However, it may be noted that addition of acac in $\mathrm{CH}_{3} \mathrm{CN}$ to MTPP in the same solvent does not quench the fluorescence of the porphyrins. Interestingly, the electronic absorption spectra of a mixture of MTPP and acac, on irradiation showed absorption bands characteristic of MTPP ${ }^{+. ' s . ~ M o r e o v e r, ~ a d d i t i o n ~ o f ~ s a c r i f i c i a l ~ d o n o r s ~ l i k e ~ E D T A ~ i n h i b i t s ~ t h e ~}$ the presence of very small quantities of isoporphyrin. Further, under similar

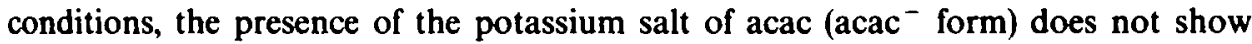
isoporphyrin formation. These observations reveal that a better nucleophile would be a radical anion relative to the anion.

The foregoing discussion indicates that the excited state electron transfer possibly provides a major pathway for fluorescence quenching. In view of the observation that $\mathrm{acac}^{-\cdot}$ has a major pathway of isoporphyrin formation, the orbital to which the excited electron is transferred is possibly the antibonding ligand $\left(\pi^{*}\right)$ or antibonding metal $t_{2 \theta}^{*}$. It seems likely that the electron from the excited state of the donor possibly enters an orbital of Fe(III) quenchers having predominatly ligand character (MLCT or LMCT). This would result in fast ligand dissociation and/or decomposition of the quencher. The $k_{Q}$ values are higher in magnitude $\left(10^{11}\right.$ to $\left.10^{12} \mathrm{~mol}^{-1} \mathrm{~s}^{-1}\right)$ in case of [Fe(mnt) $\left.{ }_{2}\right]^{-}$and $\mathrm{FeTPPCl}$. Mataga et al (1989) have observed a rapid electron transfer from $\mathrm{ZnTPP}$ to $\mathrm{FeTPPCl}$ in dimethyl formamide solution with a time constant of $30-40 \mathrm{ps}$. The magnitudes of the rates of bimolecular quenching in the micellar media, 
anionic, cationic and neutral micelles are in general lower than those observed in homogeneous media. This seems to suggest that collisional encounters control the quenching process. The binding constant values of the various quenchers with the micelles, determined by the method of Encians and Lissi (1982), are found to be low, thereby indicating negligible contribution to the rates of bimolecular quenching. The difference in the magnitudes of the $k_{Q}$ values depend on the nature of the micelle, indicating the different mobilities of charged quenchers in the micelle. The present results suggest that the excited electron transfer contributes significantly to the fluorescence quenching of the porphyrin by iron(III) coordination compounds. The validity of the suggestion should await confirmation from laser flash photolysis experiments, which are currently being pursued.

\section{Acknowledgements}

This work is financed by the Department of Atomic Energy, Trombay, Bombay. The authors are thankful to the Department of Science and Technology, Government of India, for their support. Thanks are also due to the Department of Non-Conventional Energy Sources for their financial support.

\section{References}

Averill B A, Herskovitz T, Holm R H and Ibers J A $1973 \mathrm{~J}$. Am. Chem. Soc. 953523

Averill B A and Orme W H 1979 in Metal ions in biological systems (ed.) Helmut Sigel (New York: Marcel Dekker) vol. 7

Buchler J W 1975 in Porphyrins and metalloporphyrins (ed.) K M Smith (Amsterdam: Elsevier Scientific) Demas J N and Adamson A W $1973 \mathrm{~J}$. Am. Chem. Soc. 955159

Dolphin D, Felton R H, Berg D C and Fajer J 1970 J. Am. Chem. Soc. 92743

Dorough C D, Miller J R and Huennekens F M 1951 J. Am. Chem. Soc. 734315

Encians M V and Lissi E A 1982 Chem. Phys. Lett. 9155

Fendler J H 1982 Membrane mimetic chemistry (New York: Wiley)

Fuhrhop J H and Mauzerall D 1969 J. Am. Chem. Soc. 914174

Gerloch M, Lewis J, Mabba F E and Richards A 1966 Nature (London) 212809

Hall D O and Evans M C W 1969 Nature (London) 223809

Herriott J R, Seiker L C and Johnson L H $1970 \mathrm{~J}$. Mol. Biol. 50591

Kalyanasundaram K 1987 Photochemistry in microheterogeneous systems (New York: Academic Press)

Lever A B P 1984 Inorganic electronic spectroscopy (Amsterdam: Elsevier)

Lintvedt R L and Kernitshy K K 1970 Inorg. Chem. 9491

Marcus R A 1965 J. Chem. Phys. 43679

Mataga N, Yao H and Okada T 1989 Tetrahedron 154692

Mauzerall D 1978 The porphyrins (ed.) D Dolphin (New York: Academic Press) vol. 5

Mayo S L, Ellis W R Jr, Crutchley R J and Gray H B 1986 Science 223948

McLendon G, Gaurr T, Meguire M, Simolo K, Strauch S and Taylor K 1985 Coord. Chem. Rev. 64113

Miller J R, Beitz R V and Huddleston R K 1984 J. Am. Chem. Soc. 1065051

Moriya J 1984 Bull. Chem. Soc. Jpn. 571723

Rehm D and Weller A 1970 Isr. J. Chem. 8529

Rothemund P and Mennotti A R 1941 J.Am. Chem. Soc. 63267

Rothemund P and Mennotti A R 1948 J. Am. Chem. Soc. 701808

Roy R and Mukherjee S 1987 Chem. Phys. Lett. 140210

Seely G R 1978 Photochem. Photobiol. 27639

Siggel V, Hungerbuhler H and Fuhrhop J H 1987 J. Chim. Phys. (Paris) 84 1055, and references cited therein

Weiher J F, Melby L R and Benson R E 1964 J. Am. Chem. Soc. 864329

Willigen H V and Ebersole M H 1987 J. Am. Chem. Soc. 1092299

Yang E S, Chan M S and Wahl A C 1975 J. Phys. Chem. 792049 